\title{
CAUCHY AND PÓLYA-SZEGÖ TYPE INEQUALITIES INVOLVING TWO LINEAR ISOTONIC FUNCTIONALS
}

\section{LUdMIla NikOLOVA AND SANJA VAROŠANEC}

Abstract. We consider inequalities which involve two linear isotonic functionals. We give two variants of the Cauchy inequality and few Pólya-Szegö type inequalities in which functions with variable bounds occurred. With help of these inequalities we are able to obtain a new bound for the Chebyshev difference and give some particular cases. Connections of the presented results with earlier results involving fractional integrals are also pointed out. Mathematics subject classification (2010): 26D15.

Keywords and phrases: Cauchy inequality, Chebyshev difference, fractional integral, isotonic linear functional, Pólya-Szegö type inequalities.

\section{REFERENCES}

[1] P. Agarwal, S. S. Dragomir, J. PARK, S. JAIn, q-Integral inequalities associated with some fractional q-integral operators, J. Inequal. Appl., (2015) 2015:345.

[2] P. Agarwal, J. TAriboon, S. K. Ntouyas, Some generalized Riemann-Liouville k-fractional integral inequalities, J. Inequal. Appl. (2016) 2016:122.

[3] V. L. Chinchane, D. B. PachPatte, A. B. Nale, Pólya-Szegö fractional inequality via Hadamard fractional integral, arXiv.1602.04025v1[math.CA], 12 Feb 2016.

[4] S. S. Dragomir, Congruences and inequalities of Cauchy-Buniakowski-Schwarz type, Seminar Arghiriade no. 15, Universitatea din Timişoara, Facultatea de Ştiinţe ale Naturii, Secţia Matematică, Timişoara, $1985,8 \mathrm{pp}$.

[5] S. S. Dragomir, A survey on Cauchy-Buniakowski-Schwarz type discrete inequalities, JIPAM. J. Inequal. Pure Appl. Math., 4, (2003), Issue 3, Art 63.

[6] S. S. Dragomir, Advances in Inequalities of the Schwarz, Grüss and Bessel Type in Inner Product Spaces, Nova Science Publishers, New York, 2005.

[7] S. S. Dragomir, N. T. Diamond, Integral inequalities of Grüss type via Pólya-Szegö and ShishaMond results, East Asian Math. J., 19, 1 (2003), 27-39.

[8] D. ILIŠEVIĆ, S. VAROŠ ANEC, On the Cauchy-Schwarz inequality and its reverse in semi-inner product $C^{*}$-modules, Banach J. Math. Anal., 1, (2007), 78-84.

[9] M. S. Moslehian, L.-E. Persson, Reverse Cauchy-Schwarz inequalities for positive $C^{*}$-valued sesquilinear forms, Math. Inequal. Appl., 4, (2009), 701-709.

[10] L. Nikolova, S. VAROŠAnEC, Chebyshev and Grüss type inequalities involving two linear functionals and applications, Math. Inequal. Appl., 19, 1 (2016), 127-143.

[11] L. Nikolova, S. VARošAnEC, Chebyshev-Grüss type inequalities on time scales via two linear isotonic functionals, Math. Inequal. Appl., 19, 4 (2016), 1417-1427.

[12] S. K. Ntouyas, P. Agarwal, J. Tariboon, On Pólya-Szegö and Chebyshev types inequalities involving the Riemann-Liouville fractional integral operators, J. Math. Inequal., 10, 2 (2016), 491504.

[13] J. PEČARIĆ, B. TEPEŠ, On a Grüss type inequality for isotonic linear functionals I, Nonlinear Studies, 12, (2005), 119-125.

[14] G. Pólya, G. Szegö, Problems and Theorems in Analysis, Vol. I, Translated from the German, Springer Verlag, New York-Berlin, 1972 (original version: Julius Springer Berlin 1925). 